\title{
VOISEE COMMUNICATOR: An Android Mobile Application for Hearing-impaired and Blind Communications
}

\author{
https://doi.org/10.3991/ijim.v10i4.5859 \\ Junar Arciete Landicho \\ Mindanao University of Science and Technology, Philippines
}

\begin{abstract}
Voisee Communicator is an android type mobile messaging application designed and developed for the thorough communication between two disabled people most especially the hearing-impaired and blind people. With stable and smart Eclipse IDE and the availability of different built-in libraries in java especially the tts.speech.SpeechToText and tts.speech.RecognizerIntent has been taken advantage to create custom voice command functionalities.
\end{abstract}

Creating, replying, sending and forwarding messages are among the primary and fundamental features that this study has to offer. The researchers analyzed the results of the test survey and evaluation form and proved that the application is a user friendly, efficient and accurate in delivering messages to the recipient and has the important features that the users expected.

Index Terms - hearing-impaired and blind, communication; mobile application, Text-to-Speech, Android

\section{INTRODUCTION}

What is Communication? Communication can be defined as the process of transmitting information and common understanding from one person to another (Keyton, 2011). Communication is an essential feature of humanity. It is the process of sharing our ideas, thoughts, concerns and feelings with other people and having those ideas, thoughts and feelings understood by the people whom we are talking with.

Communication - the ability to convey information to others and the ability to receive and interpret information from others - is fundamental to learning. Individuals learn about the world mostly through their senses of vision and hearing. Vision and hearing are the main sensory avenues for accessing and interacting with the world around us and for perceiving events as close or distant. When vision and hearing are reduced, even to a mild level, the losses affect the ability to communicate, develop personal relationships, and acquire concepts (Riggio and McLetchie, 2008).

Globalization is one of the main words of the 21 st century. We feel its presence in almost every field of our life: in economic, politics, culture and media (Kolosova, 2012). Accompanied by the rapid growth of information technology, android mobiles are now used widely in a variety of fields. Applications of mobile and various software in training, teaching, learning, and computer assisted instruction are a major future trend. However, most applications are designed for normal people, and are inaccessible to those who are deaf, blind, deaf-blind and people living with disabilities, unless extra adaptive tools and interfaces were designed for them.

Consequently, a current trend in high technology production is to develop adaptive tools for deaf and blind people to assist them with self-learning and personal development, and lead more independent lives (Aher, $\mathrm{Mu}-$ sale, Pagar, \& Morwal, 2014). Traditionally, technology and other interventions designed for children with disabilities were focused on strategies aimed at correcting a child's specific impairment or deficit (Wise, 2012).

Assistive Technology was designed and made for everyone. Some assistive technologies that are used by the hearing-impaired and blind are listed below:

\section{A. Braille Notetakers}

Some of the notetakers can be used in face-to-face conversation of the blind and hearing-impaired person. The hearing-impaired person types the text in the keyboard and the blind reads the text through the braille display in the device. The blind can reply through typing in the Braille keyboard the text and read in the screen by the hearing-impaired person.

\section{B. SmartTextSystem and PocketSMS}

SmartTextSystem and PocketSMS are both an Android based mobile phone system that allows blind and hearingimpaired person to send and read text messages from their phones using Morse code.

\section{Portable Communication Aid for Deaf-Blind People}

It is a two-unit system designed for face-to-face and wireless communication between a deaf-blind person and a sighted partner. It is battery operated and portable. The blind will use Braille and person without disabilities will use a normal unit.

\section{MATERIALS AND METHOdS}

\section{A. Designing of the Application}

\section{1) Analysis}

The design structure of the application is based mainly on a text messaging over telecommunication networks. The data that will be fetched for operations and functions of the application is from the device storage.

Figure 1 represents the architectural design of the Voisee Communicator. It is where the user will have to input a message into the device, and then the device having a prepaid SIM will send the inputted message to the telecommunication network. And in return, the 
telecommunication network will first check if the account is capable of sending the message, and if it does then it will forward the inputted message back to the device and the device will give the output message into the user. The application relies on the data storage of contacts and messages fetched from the device. The device which is used serves as the medium of communication between the sender and receiver.

\section{2) Context Overview}

Figure 2 shows the context overview of the application. The diagram illustrates the general process of the users involve. In the sender phase, the deaf/normal user can create text message and send it either to blind/normal user through text message which will then be converted to voice message since the blind user is a voice oriented. On the other hand, voice-oriented user which is the blind user can also send a voice message that will be converted into text message to either to blind and/or disabled user through text message that will be converted to voice or to deaf/normal user through text message. This shows the interrelatedness of both the sender and receiver.

\section{3) Detailed System Flow Design}

Figure 3 shows the entire detailed system flow of the android mobile messaging application. The user that will have this type of application will be in need of an android device with a SIM that has a load balance. In the user's device, the application has a messaging dashboard where inbox, sentbox, contacts and create message are involved. In the inbox section, the application can read and reply SMS, as well as in the Contacts section wherein it can select contact and create message. In the create message section it can add contact, add and send a message while the sentbox section can only forward a message for the hearing-impaired users.

4) Messaging Architecture Design of the Proposed Application

Figure 4 shows the architectural processes of the proposed messaging application. This figure illustrates on how Voisee Communicator application processes the processing in outputting the desired data. The message is fetched using cursor from the default inbox, sort messages from newest to oldest messages received, fetched the address (receiver's contact number) to the default contact list of the device and check if the address exists or not. If the address exists then the application will read the address and if doesn't, the application will give a keyword to the unsaved address received.

\section{B. Development of the Application}

\section{1) Software Specifications}

\section{a) Eclipse IDE}

Eclipse IDE is one of the most important software tools when it comes to developing an android application. With the development of Voisee Communicator, the researchers will use the Eclipse IDE because of its great capability to handle errors.

\section{Engine}

a) Text-To-Speech (TTS) and Speech-To-Text (STT)

With a built-in text-to-speech and speech-to-text engine, Voisee Communicator will possibly be implemented since it's an engine that converts normal language text into speech and vice versa.

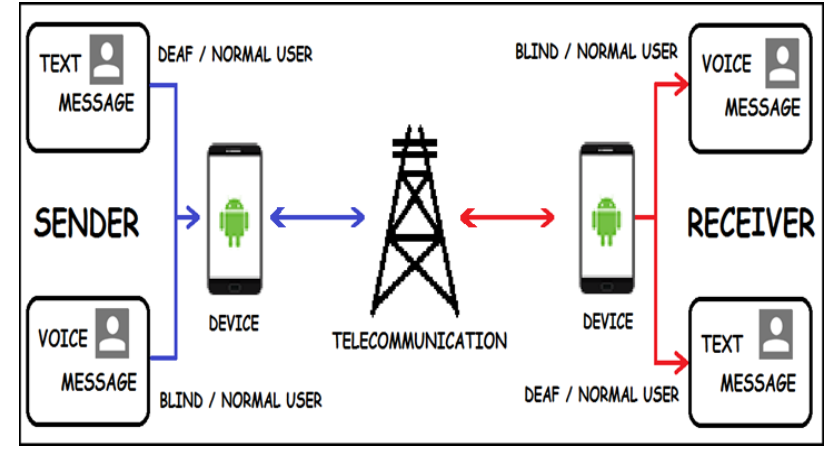

Figure 1. Architectural Design of the proposed application Voisee Communicator

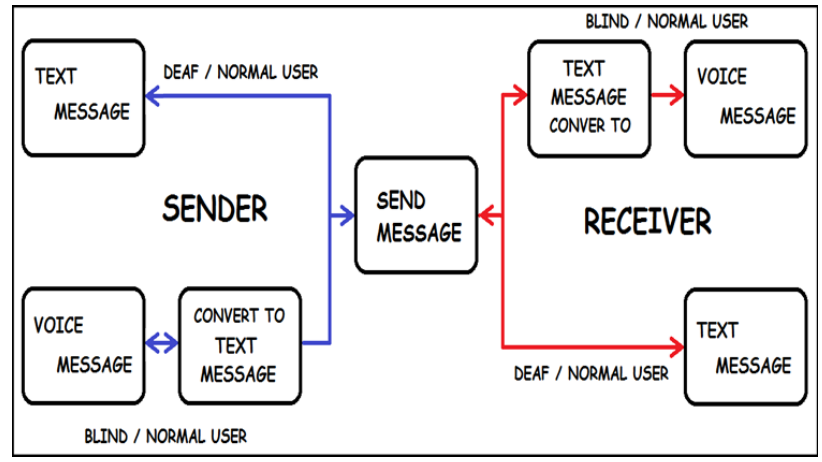

Figure 2. Proposed Context Diagram of the Application

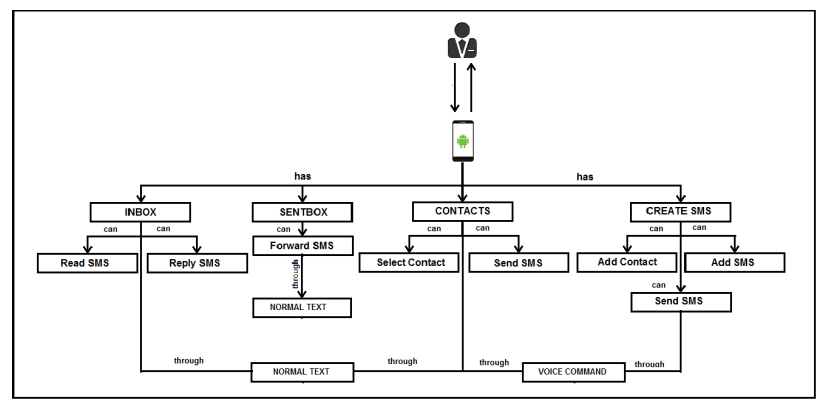

Figure 3. Proposed System Flow Design of the Application

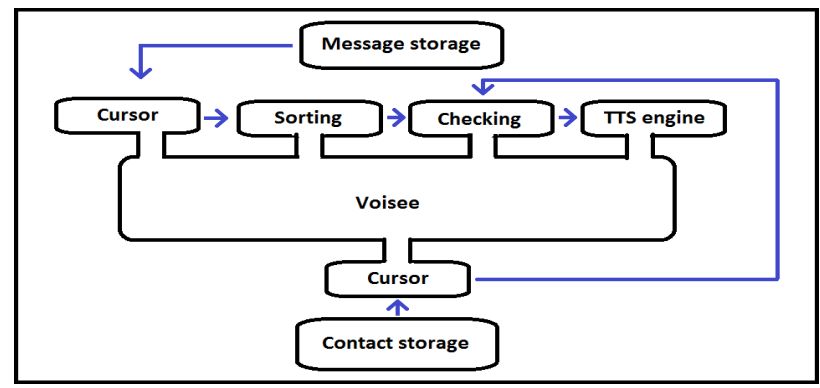

Figure 4. Messaging Architecture of Voisee Communicator

\section{2) Hardware Specifications}

\section{a) Android Smart Phone}

Smart phones have its own classification based on its brand and features. With this study, the application will be based with a phone that has an android OS with a 4.4 (KitKat) version and with a built-in text-to-speech and speech-to-text engine.

\section{b) SIM Card}

Mobile phone needs a Prepaid Subscriber Identity Module (SIM) that will be used and serves as a portable 
PAPER

VOISEE COMMUNICATOR: AN ANDROID MOBILE APPLICATION FOR HEARING-IMPAIRED AND BLIND...

memory chip for the operation on the Global System for Mobile Communication (GSM) network. SIM is important when about for testing and continuing development of the Voisee Communicator because it does hold the personal information of the account holder, including his or her phone number, address book, text messages, and other data. Addition to this, prepaid SIMs will be any of networks available depending on the subscriber as long as it has load balance of his SIM card account.

\section{c) Earphones}

Android Phones need earphones to receive radio or telephone communication and/or to listen to a message. With this device, Voisee Communicator will be able to receive accurate commands and information without being interfered by other noises in the surrounding of the user.

3) Libraries

a) Voisee Communicator incorporates the Main Libraries for Converting Texts and Recognizing Commands

import android.speech.tts.TextToSpeech; import android.speech. Recognizerlntent;

Figure 5. Screenshot of the Built-in Libraries

Figure 5 shows the main imported libraries used in Voisee Communicator development. Main libraries are imported in order to perform such necessary actions like fetching, responding, and converting. The speech.tts.TextToSpeech library executes the converting of Speech into Text and Text into Speech.Also, speech.RecognizerIntent library involves the fetching of speech and responding to the speech given.

\section{b) User Interface of the Application}

In the first phase of the first setting of the user interface of the application, Voisee Communicator's activation will be of synchronous text display and voice command sounding. With different commands instructed to the user, the application will then recognize which user is using the said application. The voice command is designed for the blind person while normal text display is for the hearingimpaired person. The blind will just tap once for Voisee Communicator's recognition and the rest of the tapping actions will be of manner for the hearing-impaired person. Moreover, in the user interface design communication process relies on the speaker sounding and normal messaging.

\section{RESUlt AND DisCUSSION}

\section{A. Designing of the Application}

\section{1) Analysis}

The following figures are various screenshots of the android mobile messaging application called Voisee Communicator.

Figure 6 shows the home interface of the Voisee Communicator with synchronous active voice command sounding for the blind and normal plain text display for the deaf. With this, Voisee Communicator will then recognize whose user is using the application. The application is only active then when the user presses the power button once and presses the volume up until the application's interface appears and talks.
Figure 7 shows the dashboard of Voisee's messaging application where inbox, sentbox, contacts and new message icons are found for easy navigation.

\section{B. Voisee Communicator's Messaging Application Interfaces for Hearing-impaired and Blind}

The hearing-impaired and blind users are using the same interface and functionality in the Voisee Communicator Messaging application. Moreover, the pop up microphone icon for voice command is intended for the blind. The figure 8 shows the inbox interface wherein the left part is the interface for the hearing-impaired and at the right part is for the blind.

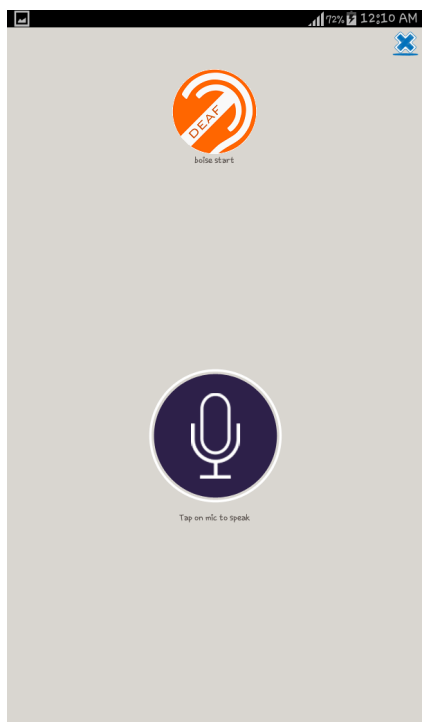

Figure 6. Voisee Communicator Home Interface

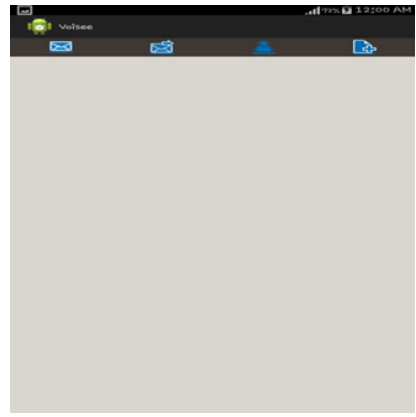

Figure 7. Voisee Communicator Messaging Dashboard

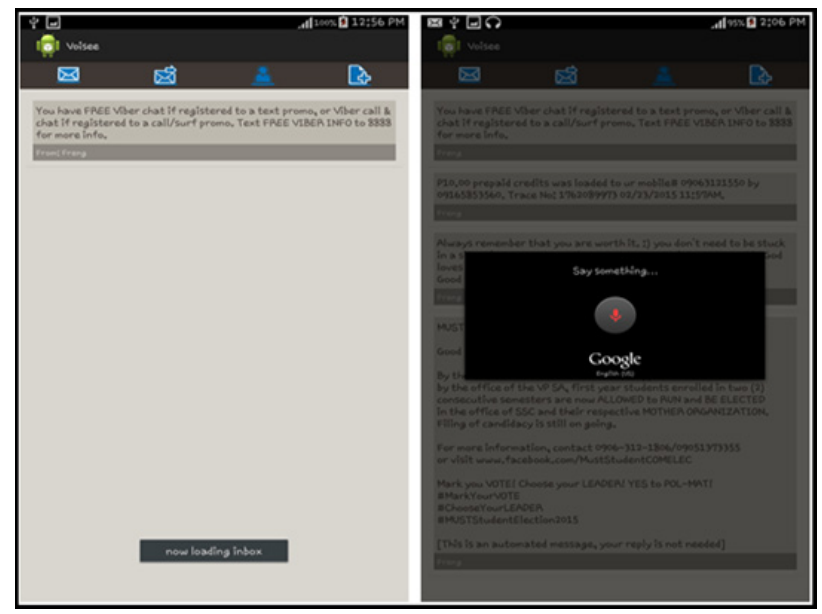

Figure 8. Screenshot of Voisee Communicator's Inbox Interface 
PAPER

VOISEE COMMUNICATOR: AN ANDROID MOBILE APPLICATION FOR HEARING-IMPAIRED AND BLIND...

In the inbox, it displays the unread message(s) and it is arranged in chronological order based on the date and time of the newest message. Thus, if the message was read already then it will be not anymore displayed in the inbox.

Figure 9 shows the sentbox where all the messages sent by the user were listed and it is sorted as first message to be sent will be in the last in the list listed. Also, the deaf user may only forward his message sent to other of his contact lists.

Figure 10 shows the Contacts section. Contacts serve as warehouse of all names with corresponding mobile number for a user to communicate with. It is arranged alphabetically for effortlessly finding name(s) to be contacted.

Figure 11 shows the section of creating a new message. The deaf user will be the one to input to what is required in creating and sending a message while the blind will be asked to add a contact name and a message to send.

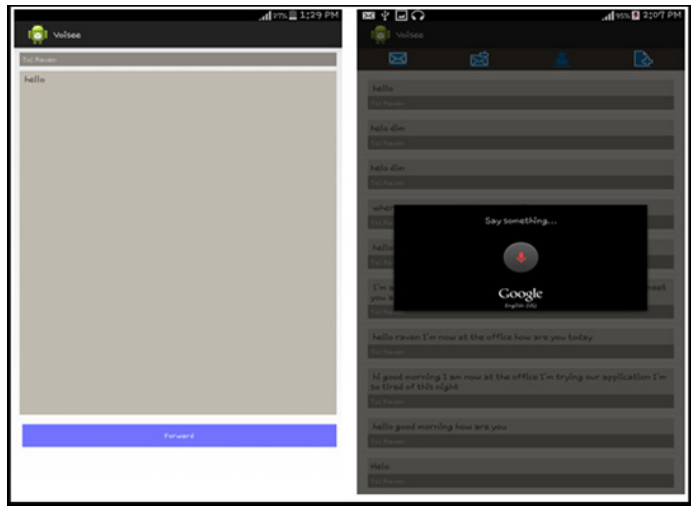

Figure 9. Screenshot of Voisee Communicator's Sentbox Interface

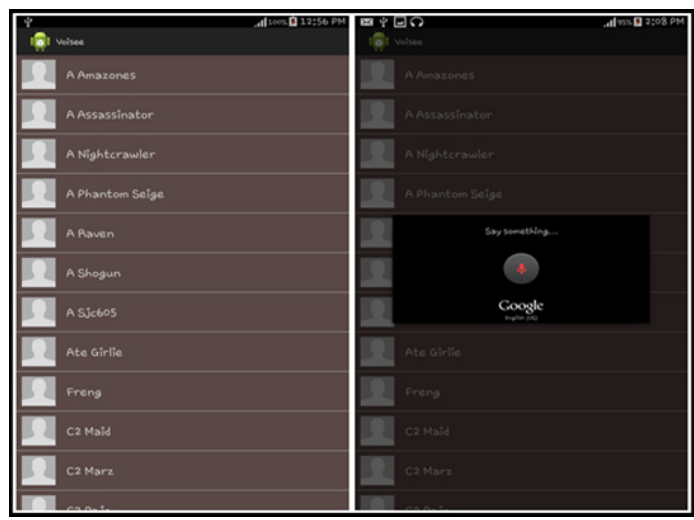

Figure 10. Screenshot of Voisee Communicator's Contacts Interface.

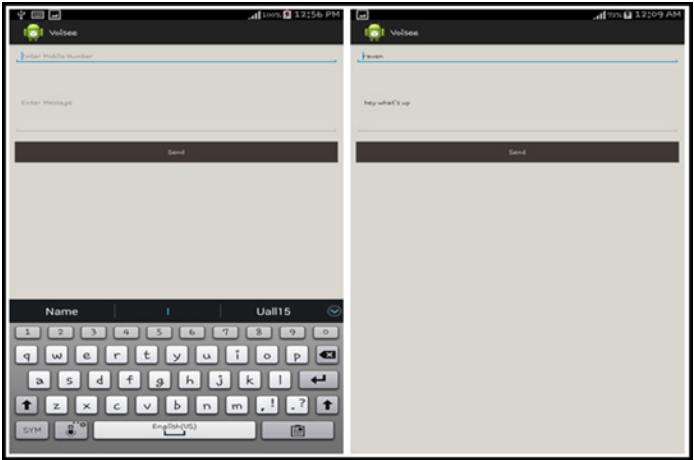

Figure 11. Screenshot of Voisee Communicator's Create New Message Interface.

\section{Evaluation of the Application}

This study has successfully conducted an application testing and survey at Cagayan de Oro National High School. The usability, performance and functionality technique was used to evaluate the mobile messaging application by the users.

\section{1) Performance Evaluation}

The performance of the application illustrates mostly the time it is recorded. The voice command delay time is set in five seconds to fetch command and load navigations.

Table 1 shows the performance evaluation results of Voisee Communicator application. After several different tests conducted, it is recommended that the operating system to be used is the 4.4 or the KitKat version. Voisee Communicator runs and operates mainly on an android device with a built-in Text-to-Speech (TTS) and Speechto-Text (STT) engine. Performance evaluation of the said application was more focus primarily with an encounter with voice aspects. In the evaluation, the duration and delay time were strictly observed to how it will correspond with an efficient reply.

\section{2) Functionality Evaluation}

The functions implemented, observed and executed in this study were based on the android normal messaging application for hearing-impaired and person without disabilities. While in the blind is the voice command implementation.

Table II shows the functionality evaluation result of Voisee Communicator where every navigation implemented in the application was manually tested and proven by the hearing-impaired, blind and even people with disabilities respondents.

TABLE I.

PERFormanCE EVALUATION RESUlt

\begin{tabular}{|c|c|c|c|c|c|c|c||}
\hline $\begin{array}{c}\text { Trs/sT Engine } \\
\text { Compatibility }\end{array}$ & $\begin{array}{c}\text { RAM } \\
\text { Usage }\end{array}$ & CPU Usage & \multicolumn{2}{|c|}{ Duration (second) } & \multicolumn{2}{|c||}{ Delay (second) } & $\begin{array}{c}\text { Reading } \\
\text { Message } \\
\text { (millisecond) }\end{array}$ \\
\hline & & & Fetch & Response & Navigation & Commands & $\begin{array}{c}\text { Num. Of words } \\
\text { multiply by } \\
500 \mathrm{~ms}\end{array}$ \\
\hline $\begin{array}{c}\text { Built-in } \\
\text { (Required) }\end{array}$ & $9.17 \mathrm{MB}$ & $0.01 \%$ & $4 \mathrm{~s}$ max. & $2 \mathrm{~s}$ & $5 \mathrm{~s}$ & $5 \mathrm{~s}$ & \\
\hline
\end{tabular}

TABLE II.

FUNCTIONALITY EVALUATION RESULT

\begin{tabular}{cll}
\hline NAVIGATIONS & REMARKS \\
\hline $\begin{array}{c}\text { Inbox } \\
\bullet \quad \text { Read Message }\end{array}$ & Passed \\
$\bullet \quad$ Reply Message & Passed \\
$\begin{array}{c}\text { Create Message } \\
\bullet \quad \text { Add Contact }\end{array}$ & Passed \\
$\bullet \quad$ Add Message & Passed \\
• & Send Message & Passed \\
Sentbox & & \\
$\bullet$ & Forward Message & Passed \\
Contacts & & \\
$\bullet$ & Select Contact & Passed \\
$\bullet$ & Send Message & Passed \\
\hline Overall & & Passed
\end{tabular}


Hearing-impaired respondents were asked to evaluate on how the activation of the application performs throughout the messaging actions they executed through tapping. Likewise, with the blind respondent they would execute the messaging actions through voice command. The evaluation was measured through a passed or failed remarks and an overall remark of passed.

\section{3) Usability Evaluation}

Table 3 shows the result of the usability evaluation of the mobile applications. Ten (10) blind, ten (10) hearingimpaired and ten (10) persons without disabilities evaluated the application. Each criterion has the following rating scale: 1-bad, 2-poor, 3-moderate, 4-good and 5excellent.

In the communication quality criteria, the respondent satisfied to the conversion of speech to text is accurate and convenient to use. Both blind and hearing-impaired respondents agreed that the application can help independency when communicating others. In the response quality criteria, the application can successfully send and receive message with accurate and clarity message. In the application quality critieria, the respondent believe it is a user friendly type because of its simple instructions to follow, easy to used commands and have navigation facilities that support the needs in doing communication.

TABLE III

USABILITY EVALUATION RESULT

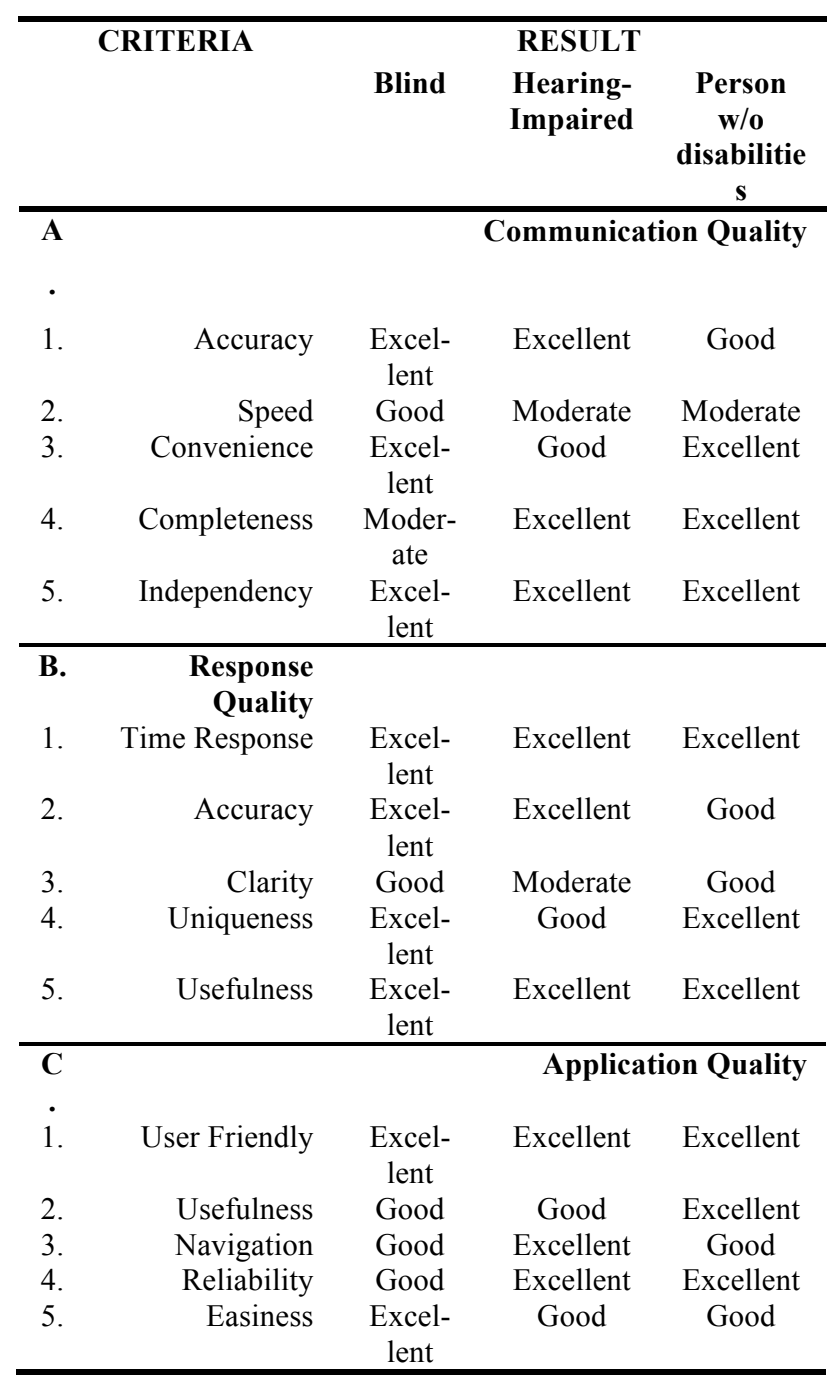

\section{CONCLUSION AND RECOMMENDATIONS}

\section{A. Conclusion}

The Voisee Communicator has undergone tests to know whether the problems of the communication between the two disabled people have been resolved and the objectives of the study have been met. And gather information on the outlook of the user about the design and functionality of the proposed application.

The android mobile messaging app has its own benefit but lacks interactive functionalities most importantly for the blind. Blind people hesitant to use an android phone and even android messaging application because of highly sensitive in touching surface. The new messaging application was made to provide a better and friendly user interface making it more beneficial to the users most especially the hearing-impaired and blind. Voisee Communicator mainly made to help and aid the communication between two - hearing-impaired and blind people and also to prove the efficiency and clarity of the message they will convey with each other. After conducting several test, the researchers found out that the application is efficient and easy enough in managing their daily independent communication with others.

\section{B. Recommendations}

Referring to the results on the dry run of the android based mobile messaging application; the proponents can say that the proposed application lacks some functions that can cater all the needs of the respondents. Therefore, it is highly recommended that the application will continuously be modified to best fit the needs of the said disabled people.

Mobile messaging application recommendations are as follows:

1. Fetching of voice commands should be faster.

2. The application should understand other languages.

3. Improving the user interface of the android messaging application.

4. The application should have other features wherein the user may use Wi-fi or internet connection in lieu of a zero load balance in their account.

5. Allows the application to have permission to perform necessary actions like to update and delete.

\section{FINANCIAL DISCLOSURE}

There is no conflict of interest.

\section{FUNDING/SUPPORT}

No financial assistance was obtained from any organization or company. The project was funded by the institution itself.

\section{REFERENCES}

[1] Aher, A., Musale, K., Pagar, S., Morwal, S.(2014). Implementation of Smart Mobile App for Blind \& Deaf Person Using Morse Code. International Journal of Research in Advent Technology, Vol.2, (No.2, February 2014), 151-154.

[2] April A. Kedrowicz, Julie L. Taylor. (2013). ENGINEERING COMMUNICATION AND THE GLOBAL WORKPLACE Preparing Professionals And global citizens. International Professional Communication Journal, 87-88.

[3] Azenkot, S., \& Fortuna, E. (2010). Improving Public Transit Usability for Blind and Deaf-Blind People by Connecting a Braille 
PAPER

VOISEE COMMUNICATOR: AN ANDROID MOBILE APPLICATION FOR HEARING-IMPAIRED AND BLIND...

Display to a Smartphone. Orlando, Florida, USA. http://dx.doi.org/10.1145/1878803.1878890

[4] Bernatowicz, A., \& Iwanski, R. (2012). Informational Marginalization In Digital Media Among People Over 45 Years Of Age In Poland. Journal Of International Scientific Publications: Media and Mass Communication, 3.

[5] Demeter, M. (2012). On Analysis and its Role in Communication Theories. KOME - An International Journal of Pure Communication Inquiry, 38. http://dx.doi.org/10.17646/kome. $\underline{2012.14}$

[6] Heckendorf, S. (2009). Assistive Technology For Individuals Who Are Deaf Or Hard Of Hearing. In Assessing Students' Needs for Assistive Technology (p. 430). 448 East High Street Milton, WI 53563.

[7] Jackson, R. M. (2012). Audio-Supported Reading for Students who are Blind or Visually Impaired. 40 Harvard Mills Square, Suite 3.

[8] Kelly, S. M., \& Smith, D. W. (2011). The Impact of Assistive Technology on the Educational Performance of Students with Visual Impairements: A Synthesis of the Research. Joumal of Visual Impairment \& Blindness, 1-5.

[9] Keyton, J. (2011). Communication and organizational culture: A key to understanding work experience. Thousand Oaks, CA: Sage

[10] Kolosova, A (2012). Globalization, Culture, And The Role Of Media. Journal of International Scientific Publications: Media and Mass Communication, 69,72.

[11] Lunenburg, F. C. (2010). Communication: The Process, Barriers, And Improving Effectiveness. Schooling, 2-3.

[12] Lutterer, W. (2007). The Two Beginnings of Communication Theory. Kybernetes: The International Journal of Systems \& Cybernetics, 36. Jg., $\quad H . \quad 7 / 8, \quad S . \quad 1022-1025, \quad 2-3$. http://dx.doi.org/10.1108/03684920710777793

[13] Ogbomo, M. O., \& Ogbomo, E. F. (2008). Importance of Information and Communication Technologies (ICTs) in Making a Heathy Information Society: A Case Study of Ethiope East Local Government Area of Delta State, Nigeria. Library Philosophy and Practice, 3.

[14] Perron, B. E., Taylor, H. O., Glass, J. E., \& Margerum-Leys, J. (2010). Information and Communication Technologies in Social Work. Advances in Social Work, 1-2.
[15] Riemer, M. J. (2007). Communication Skills for the 21st Century Engineer. Global Journal of Engineering Education, 89.

[16] Riggio M. and McLetchie B. (2008). Issues and a Practices In Deafblindness: Educational Service Guidelines(p. 4). Perkins School for the Blind.

[17] Saj, H. E., \& Sarraf, C. M. (2012). Media Influence On Working Class Women Social Values In Abu Dhabi. Journal Of International Scientific Publications: Media and Mass Communication, 40.

[18] Smarandache, F., \& Vladutescu, S. (2014). Towards A Practical Communication Intervention, 244.

[19] Su, M.-C., Chen, C.-Y., Su, S.-Y., Chou, C.-H., Hsiu, H.-F., \& Wang, Y.-C. (2001). Portable Communication Aid for Deaf-blind People. Computing \& Control Engineering Journal, 1-3.

[20] Uldall, T. S., Astell, A. J., Ellis, M. P., Olesen, H. H., Edberg, P. O., \& Westerholm, B. (2012). A Good Senior Life with Dual Sensory Loss. Denmark: Nordic Centre for Welfare and Social Issues.

[21] Wise, P. H. (2012). Emerging Technologies and Their Impact on Disability. In Children with Disabilities (pp. 171-182). Princeton. http://dx.doi.org/10.1353/foc.2012.0002

\section{AUTHOR}

J A Landicho is a BS Computer Science at Caraga State University, Butuan City, Philippines last 2006. He earned his Master in Information Technology at Mindanao University of Science and Technology, Cagayan de Oro City, Philippines last 2012. He is a faculty member in the Department of Information Technology of the Mindanao University of Science and Technology, Philippines.. His research interest is in the field of database system, mobile application, image and sound processing (e-mail: junarlandicho@must.edu.ph).

Submitted 22 May 2016. Published as resubmitted by the author 23 September 2016. 